\title{
Efficacy of subcutaneous granulocyte colony- stimulating factor infusion for treating thin endometrium
}

\author{
Kaberi Banerjee, Bhavana Singla, Priyanka Verma \\ Advance Fertility and Gynaecology Centre, New Delhi, India
}

Objective: This study was conducted to assess the efficacy of subcutaneous granulocyte colony-stimulating factor (G-CSF) for treating thin endometrium.

Methods: Data from 88 infertile women with thin endometrium $(<7 \mathrm{~mm})$ aged 23 to 40 years were evaluated retrospectively over a period of 1 year. In group 1, subcutaneous infusion of G-CSF $(300 \mu \mathrm{g} / \mathrm{mL})$ was administered to 44 women along with other supplemental treatments. If the lining did not exceed $7 \mathrm{~mm}$ within 72 hours, a second infusion was administered. In group 2, which also had 44 women, only estradiol valerate and sildenafil were administered, while subcutaneous G-CSF infusion was not. Embryo transfers were performed once the lining exceeded $7.5 \mathrm{~mm}$. The efficacy of G-CSF was evaluated by assessing the thickness of the endometrium before embryo transfer, pregnancy rates, and clinical pregnancy rates.

Results: There were no differences between the groups regarding demographic variables, egg reserves, sperm parameters, the number of embryos transferred, and embryo quality. The pregnancy rate was significantly higher in group 1 (60\%, 24 of 40 cases) than in group $2(31 \%$, 9 of 29 cases) $(p<0.001)$. The clinical pregnancy rate was also significantly higher in group $1(55 \%)$ than in group $2(24 \%)(p<0.001)$.

Conclusion: Subcutaneous G-CSF infusion improved the thickness of the endometrium when it was thin. To the best of our knowledge, this is the first documented study to clearly demonstrate the benefits of subcutaneous G-CSF infusion for treating thin endometrium.

Keywords: Granulocyte colony-stimulating factor; Infusion; Subcutaneous; Thin endometrium

\section{Introduction}

Despite recent technological developments, in vitro fertilization (IVF) is still not a surefire solution to infertility, and implantation failure remains a major problem that leads to unsuccessful IVF. Successful implantation depends on many factors such as egg quality, sperm quality, embryo quality, the receptivity of the endometrium, and the quality of the embryo transfer technique [1]. The endometrium is

Received: July 17, 2021 - Revised: October 14, 2021 · Accepted: November 13, 2021 Corresponding author: Bhavana Singla

Advance Fertility and Gynaecology Centre, 6, Ring Rd, Lajpat Nagar 4, New Delhi 110049 , India

Tel:+91-98-7109-6615 Fax:+91-93-5000-5568 E-mail: drbhavanasingla@gmail.com

This is an Open Access article distributed under the terms of the Creative Commons Attribution Non-Commercial License (http://creativecommons.org/licenses/by-nc/4.0/) which permits unrestricted non-commercial use, distribution, and reproduction in any medium, provided the original work is properly cited. very important for embryo implantation, and endometrial thickness is a marker of endometrial receptivity. It is used as a prognostic factor in embryo transfers [2,3]. Adequate endometrial thickness is essential for a successful pregnancy. A thin endometrium adversely affects the rate of successful reproduction using IVF. The definition of a thin endometrium varies across studies, but it is generally defined as an endometrium of $<7 \mathrm{~mm}$ on the day of human chorionic gonadotropin (hCG) injection during fresh embryo transfer cycles and $<7-8$ $\mathrm{mm}$ on the day that progesterone supplementation is added for frozen-thawed cycles [4-6]. The reported prevalence of thin endometrium in assisted reproductive technology patients is between $1.5 \%$ and $9.1 \%[6,7]$.

There are various treatment regimens that aim to improve endometrial thickness, but only some have been found to yield actual improvements. Granulocyte colony-stimulating factor (G-CSF) has recently been suggested as a possible treatment for thin endometrium 
$[8,9]$. G-CSF is a glycoprotein that combines growth factor and cytokine activities. It is secreted in various tissues, including reproductive tissues such as the endothelium and ovarian follicles, as well as immunocytes (e.g., macrophages) [10]. It stimulates neutrophilic granulocyte proliferation and differentiation and acts on decidual macrophages, affecting implantation. It also recruits dendritic cells, promotes Th- 2 cytokine secretion, activates $T$ regulatory cells, and stimulates various proangiogenic effects that also affect implantation [11]. In most studies, intrauterine infusions of G-CSF were administered to improve the uterine lining $[8,9]$. However, G-CSF can also be administered subcutaneously to improve the thickness of the endometrium [12]. In this study, we examined the efficacy of subcutaneous G-CSF infusion for treating thin endometrium.

\section{Methods}

The study was conducted at the Advance Fertility and Gynaecology Centre in New Delhi, India, from January 2019 to December 2019. Patients with primary and secondary infertility between the ages of 23 to 40 years were included in the study. In total, 88 infertile women were examined, and the inclusion criteria were as follows. (1) A history of at least one previous IVF failure and thin endometrium ( $<7 \mathrm{~mm}$ ) 15 to 18 days into a regular 28-to-30-day cycle. (2) A history of canceled embryo transfer due to thin endometrium $(<7 \mathrm{~mm}$ ) on the day of hCG injection. (3) A history of thin endometrium ( $<7 \mathrm{~mm}$ ) 12 or 13 days after beginning estrogen supplementation ( 6 to $10 \mathrm{mg} /$ day).

In group 1, 44 women undergoing either fresh or frozen cycles were examined. Those undergoing fresh cycles received subcutaneous injections of G-CSF ( $300 \mu \mathrm{g} / \mathrm{mL}$ ) starting on day 7 of hormonal injection along with oral estradiol valerate (4 to $6 \mathrm{mg} /$ day) to increase endometrial thickness and vaginal sildenafil (50 mg/day) to increase uterine blood flow. Those undergoing frozen cycles received oral estradiol valerate ( $6 \mathrm{mg} /$ day) starting on day 2 of the menstrual cycle and subcutaneous injections of G-CSF $(300 \mu \mathrm{g} / \mathrm{mL})$ starting on the 7th day after beginning medication, along with an increased dose of oral estradiol valerate ( $10 \mathrm{mg} /$ day) and vaginal sildenafil (50 $\mathrm{mg} /$ day). If the endometrial thickness did not exceed $7.5 \mathrm{~mm}$ within 72 hours, a second injection was given, and the other supplements were continued. A final G-CSF injection was administered on the day of oocyte retrieval for those undergoing fresh cycles and on the day that progesterone was added for those undergoing frozen cycles.

In group 2, which also included 44 women, estradiol valerate and sildenafil were given to those undergoing fresh and frozen cycles like in group 1; however, subcutaneous G-CSF infusion was not administered (Figure 1). Embryo transfer was performed only when the endometrial thickness exceeded $7.5 \mathrm{~mm}$ and there was subjective improvement in the echotexture of the lining based on two-dimen- sional ultrasonography. The efficacy of G-CSF was evaluated by assessing endometrium thickness before embryo transfer, the pregnancy rate, and the clinical pregnancy rate.

\section{Results}

There were no significant differences between the groups regarding demographic variables, ovarian reserve (donor eggs were used in instances of low ovarian reserve), sperm parameters, number of embryos transferred, and embryo quality (Table 1).

In group 1, the embryo transfer was cancelled in four cases, and in group 2, there were 15 cancellations due to thin endometrium even after treatment, showing statistical significance $(p=0.008)$. The pregnancy rate was significantly higher in group 1 (60\%, 24 out of 40 cases) than in group 2 (31\%, 9 out of 29 cases) $(p<0.001)$. The clinical pregnancy rate was also significantly higher in group $1(55 \%)$ than in group $2(24 \%)(p<0.001)$ (Table 2).

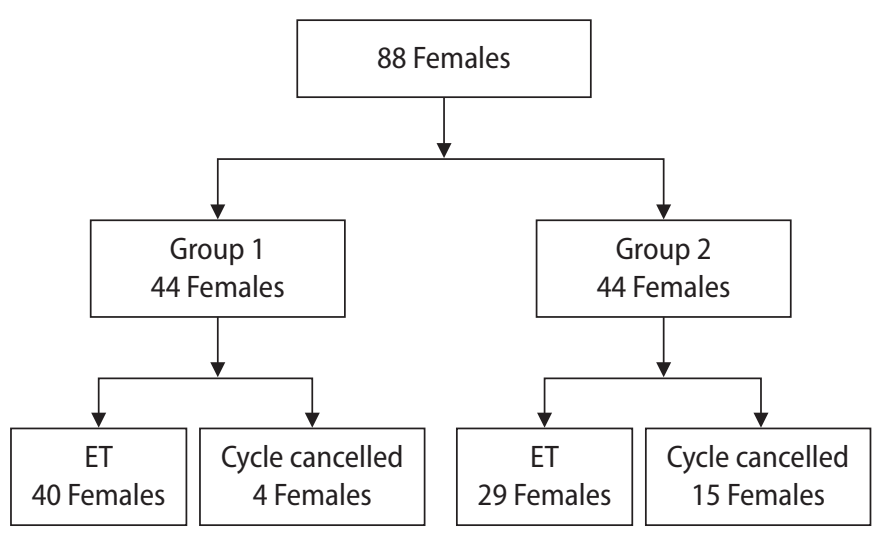

Figure 1. Flow diagram of study design. ET, embryo transfer.

Table 1. Demographic profile

\begin{tabular}{lccc}
\hline Parameter & $\begin{array}{c}\text { Group 1 } \\
\text { (with G-CSF) }\end{array}$ & $\begin{array}{c}\text { Group 2 } \\
\text { (without G-CSF) }\end{array}$ & $p$-value \\
\hline Number of patients & 44 & 44 & \\
Age (yr) & 31.6 & 31.8 & 0.12 \\
Length of infertility (yr) & 4.3 & 5 & 0.47 \\
Primary & 25 & 23 & 1.12 \\
Secondary & 19 & 21 & 1.12 \\
BMl (kg/m ${ }^{2}$ ) & 20.8 & 21 & 0.22 \\
Antral follicle count & 8.4 & 8.1 & 0.47 \\
AMH level (ng/mL) & 2.7 & 2.8 & 0.17 \\
\hline
\end{tabular}

G-CSF, granulocyte colony-stimulating factor; BMl, body mass index; $\mathrm{AMH}$, anti-Müllerian hormone. 
Table 2. IVF outcome

\begin{tabular}{lccc}
\hline Parameter & $\begin{array}{c}\text { Group 1 } \\
\text { (with G-CSF, } \mathrm{n}=44)\end{array}$ & $\begin{array}{c}\text { Group 2 (without } \\
\text { G-CSF, } \mathrm{n=44)}\end{array}$ & p-value \\
\hline ET $>7.5 \mathrm{~mm}$ & 40 & 29 & $<0.001$ \\
Cycle cancelled & 4 & 15 & $<0.001$ \\
Pregnancy rate & $24 / 40(60)$ & $9 / 29(31)$ & $<0.001$ \\
Clinical pregnancy rate & $22 / 40(55)$ & $7 / 29(24)$ & $<0.001$ \\
\hline
\end{tabular}

Values are presented as number or number (\%).

IVF, in vitro fertilization; G-CSF, granulocyte colony-stimulating factor; ET, endometrial thickness.

\section{Discussion}

Endometrial thickness is a marker of endometrial receptivity and is important for embryo implantation. Various treatments have been suggested in studies for improving endometrial thickness, but some remain unproven due to the limited number of subjects. In cases of thin endometrium, embryos from that particular cycle are frozen, and the endometrium is prepared again in order to perform an optimal embryo transfer.

G-CSF is an emerging treatment method for thin endometrium that has shown promising results. In our study, we assessed the effect of subcutaneous G-CSF infusion for improving the thickness of the endometrium. Zhang et al. [13] conducted a meta-analysis of 10 randomized control studies involving 1,016 IVF embryo transfer cycles, found that treatment with G-CSF infusion improved clinical outcomes after embryo transfer when performed using both local and systematic infusion, especially in cases of repeated implantation failure, and concluded that further randomized control trials are needed to investigate the efficacy of G-CSF infusion for patients with thin endometrium [13]. Most previous studies assessed the intrauterine effects of G-CSF infusion on thin endometrium. Gleicher et al. [8] and Kunicki et al. [9] found that intrauterine G-CSF infusion was effective for treating chronically thin endometrium and that the thickness of the endometrium significantly increased after G-CSF infusion, though it did not vary between conception and non-conception cycles. Both studies measured an ongoing clinical pregnancy rate of approximately 19\%. Barad et al. [14] reported that G-CSF did not affect endometrial thickness, implantation rates, or clinical pregnancy rates among healthy IVF patients with normal endometrium or older IVF patients. Davari-Tanha et al. [15] conducted a double-blind placebo randomized control trial with 100 subjects in whom $300-\mu \mathrm{g}$ intrauterine infusions of G-CSF were performed, and it was found that the infusions may have increased the chemical pregnancy and implantation rates of patients with recurring implantation failure; however, the clinical pregnancy rate and miscarriage rate were not affected. Very few studies have assessed the efficacy of subcutaneous G-CSF infusion for treating repeated implantation failure. Aleyasin et al. [12] found that single-dose systemic subcutaneous G-CSF infusion before implantation significantly increased the rates of successful IVF, implantation, and pregnancy (44.6\%) in infertile women with repeated IVF failure. Scarpellini and Sbracia $[16,17]$ found that G-CSF infusion might be effective for treating unexplained recurrent miscarriage and repeated implantation failure. Kamath et al. [18], in a Cochrane review, expressed uncertainty about the role of G-CSF for treating thin endometrium, and stated that the quality of the evidence suggesting that G-CSF infusion may improve the clinical pregnancy rate in women who have experienced two or more IVF failures was low. Zhao et al. [19] found that subcutaneous G-CSF infusion resulted in significantly higher pregnancy and implantation rates compared to the control group, whereas G-CSF administered via local uterine infusion had no beneficial effects on pregnancy and implantation rates in cases of recurrent IVF failure. Another recent study found that G-CSF infusion improved endometrial thickness regardless of whether the intrauterine or subcutaneous method was used. Although the intrauterine method showed slightly better results than the subcutaneous method, the degree of improvement was not statistically significant. Hence, the subcutaneous method can still be offered to patients, making it a viable option for performing G-CSF infusions to improve endometrial thickness and flow in patients with thin endometrium undergoing an embryo transfer [20].

Our study demonstrated the beneficial effects of G-CSF infusion using the subcutaneous method for treating thin endometrium, which is easier to administer than the intrauterine infusion method and does not require any extra steps. Subcutaneous G-CSF infusion was found to increase endometrial thickness and pregnancy rates in the study subjects. Other studies have examined the effects of subcutaneous G-CSF infusion in cases of recurrent IVF failure and unexplained recurrent miscarriages, but its effects on thin endometrium have not yet been established. Larger cohort studies are required in the future to further examine the effects of subcutaneous G-CSF infusion on thin endometrium. To the best of our knowledge, this is the first documented study to clearly demonstrate the beneficial effects of subcutaneous G-CSF infusion on thin endometrium.

\section{Conflict of interest}

No potential conflict of interest relevant to this article was reported.

\section{ORCID}

$\begin{array}{ll}\text { Kaberi Banerjee } & \text { https://orcid.org/0000-0002-3744-7004 } \\ \text { Bhavana Singla } & \text { https://orcid.org/0000-0003-3151-9334 } \\ \text { Priyanka Verma } & \text { https://orcid.org/0000-0003-0011-3593 }\end{array}$ 


\section{Author contributions}

Conceptualization: KB. Data curation: PV. Formal analysis: BS. Methodology: BS. Project administration: KB. Visualization: BS. Writing-original draft: BS. Writing-review \& editing: BS.

\section{References}

1. Eftekhar M, Hosseinisadat R, Baradaran R, Naghshineh E. Effect of granulocyte colony stimulating factor (G-CSF) on IVF outcomes in infertile women: an RCT. Int J Reprod Biomed 2016;14:341-6.

2. Jimenez PT, Schon SB, Odem RR, Ratts VS, Jungheim ES. A retrospective cross-sectional study: fresh cycle endometrial thickness is a sensitive predictor of inadequate endometrial thickness in frozen embryo transfer cycles. Reprod Biol Endocrinol 2013;11:35.

3. Check JH, Nowroozi K, Choe J, Dietterich C. Influence of endometrial thickness and echo patterns on pregnancy rates during in vitro fertilization. Fertil Steril 1991;56:1173-5.

4. El-Toukhy T, Coomarasamy A, Khairy M, Sunkara K, Seed P, Khalaf $Y$, et al. The relationship between endometrial thickness and outcome of medicated frozen embryo replacement cycles. Fertil Steril 2008;89:832-9.

5. Kasius A, Smit JG, Torrance HL, Eijkemans MJ, Mol BW, Opmeer $\mathrm{BC}$, et al. Endometrial thickness and pregnancy rates after IVF: a systematic review and meta-analysis. Hum Reprod Update 2014; 20:530-41.

6. Wu Y, Gao X, Lu X, Xi J, Jiang S, Sun Y, et al. Endometrial thickness affects the outcome of in vitro fertilization and embryo transfer in normal responders after GnRH antagonist administration. Reprod Biol Endocrinol 2014;12:96.

7. Aydin T, Kara M, Nurettin T. Relationship between endometrial thickness and in vitro fertilization-intracytoplasmic sperm injection outcome. Int J Fertil Steril 2013;7:29-34.

8. Gleicher N, Kim A, Michaeli T, Lee HJ, Shohat-Tal A, Lazzaroni E, et al. A pilot cohort study of granulocyte colony-stimulating factor in the treatment of unresponsive thin endometrium resistant to standard therapies. Hum Reprod 2013;28:172-7.

9. Kunicki M, Lukaszuk K, Woclawek-Potocka I, Liss J, Kulwikowska P, Szczyptanska J. Evaluation of granulocyte colony-stimulating factor effects on treatment-resistant thin endometrium in women undergoing in vitro fertilization. Biomed Res Int 2014;2014:913 235.

10. Gleicher N, Vidali A, Barad DH. Successful treatment of unresponsive thin endometrium. Fertil Steril 2011;95:2123.

11. Rutella S, Zavala F, Danese S, Kared H, Leone G. Granulocyte colony-stimulating factor: a novel mediator of T cell tolerance. J Immunol 2005;175:7085-91.

12. Aleyasin A, Abediasl Z, Nazari A, Sheikh M. Granulocyte colony-stimulating factor in repeated IVF failure, a randomized trial. Reproduction 2016;151:637-42.

13. Zhang L, Xu WH, Fu XH, Huang QX, Guo XY, Zhang L, et al. Therapeutic role of granulocyte colony-stimulating factor (G-CSF) for infertile women under in vitro fertilization and embryo transfer (IVF-ET) treatment: a meta-analysis. Arch Gynecol Obstet 2018; 298:861-71.

14. Barad DH, Yu Y, Kushnir VA, Shohat-Tal A, Lazzaroni E, Lee HJ, et al. A randomized clinical trial of endometrial perfusion with granulocyte colony-stimulating factor in in vitro fertilization cycles: impact on endometrial thickness and clinical pregnancy rates. Fertil Steril 2014;101:710-5.

15. Davari-Tanha F, Shahrokh Tehraninejad E, Ghazi M, Shahraki Z. The role of G-CSF in recurrent implantation failure: a randomized double blind placebo control trial. Int J Reprod Biomed 2016;14:737_ 42.

16. Scarpellini F, Sbracia M. G-CSF treatment improves IVF outcome in women with recurrent implantation failure in IVF. J Reprod Immunol 2012;94:103.

17. Scarpellini F, Sbracia M. Granulocyte colony-stimulating factor for the treatment of recurrent miscarriage. J Reprod Immunol 2011; 90:158-9

18. Kamath MS, Kirubakaran R, Sunkara SK. Granulocyte-colony stimulating factor administration for subfertile women undergoing assisted reproduction. Cochrane Database Syst Rev 2020;1: CD013226.

19. Zhao J, Xu B, Xie S, Zhang Q, Li YP. Whether G-CSF administration has beneficial effect on the outcome after assisted reproductive technology?: a systematic review and meta-analysis. Reprod Biol Endocrinol 2016;14:62.

20. Singal S, Sharma RK, Ahuja N. GCSF in patients with thin endometrium: subcutaneous or intrauterine? Fertil Sci Res 2020;7:43-8. 